\title{
Dielectric catastrophe at the Mott transition
}

\author{
C. Aebischer, D. Baeriswyl, and R. M. Noack \\ Institut de Physique Théorique, Université de Fribourg, CH-1700 Fribourg, Switzerland
}

\begin{abstract}
We study the Mott transition as a function of interaction strength in the half-filled Hubbard chain with next-nearest-neighbor hopping $t^{\prime}$ by calculating the response to an external electric field using the Density Matrix Renormalization Group. The electric susceptibility $\chi$ diverges when approaching the critical point from the insulating side. We show that the correlation length $\xi$ characterizing this transition is directly proportional to fluctuations of the polarization and that $\chi \sim \xi^{2}$. The critical behavior shows that the transition is infinite-order for all $t^{\prime}$, whether or not a spin gap is present, and that hyperscaling holds.
\end{abstract}

PACS numbers: 71.10.Fd, 71.30.+h, 75.40.Cx

A material's response to an applied electric field characterizes whether it is a metal or an insulator. One such response is the static electrical conductivity at zero temperature, which is finite for a metal (or infinite for an ideal conductor), but vanishes for an insulator [1]. The conductivity can therefore be used to probe the metalinsulator transition from the metallic side. A complementary quantity is the dielectric response to an electric field, the electric susceptibility, $\chi$. This quantity is expected to diverge (for a continuous transition) when the transition is approached from the insulating side and to remain infinite in the metallic phase. This phenomenon, termed "dielectric catastrophe" by Mott [2], has been reported for doped silicon [3].

One possible origin of insulating behavior is the local Coulomb repulsion between electrons. This "Mott phenomenon" [1] leads to a metal-insulator transition which occurs either as the electron density, $n$, is varied for fixed electron-electron interaction strength or as a function of interaction strength at fixed electron density [2.5]. In this letter, we concentrate on the transition as a function of interaction strength for fixed electron density. Experimentally, such a transition can be induced by applying isostatic or chemical pressure.

The prototype model for the Mott transition is the single-band Hubbard model with purely local interaction, whose Hamiltonian is

$$
\hat{H}=-\sum_{i j \sigma} t_{i j} \hat{c}_{i \sigma}^{\dagger} \hat{c}_{j \sigma}+U \sum_{i} \hat{n}_{i \uparrow} \hat{n}_{i \downarrow}
$$

where $\hat{c}_{i \sigma}^{\dagger}$ creates an electron of spin $\sigma$ at site $i$ and $\hat{n}_{i \sigma} \equiv \hat{c}_{i \sigma}^{\dagger} \hat{c}_{i \sigma}$. The hopping matrix elements $t_{i j}$ are shortranged. At half-filling, $n=1$, the Hamiltonian (11) maps onto a Heisenberg model with couplings $J_{i j}=4 t_{i j}^{2} / U$ for $U \rightarrow \infty$ and is thus insulating, while at $U=0$, it describes a perfect metal. Therefore, a Mott transition must occur at some $U_{c} \geq 0\|6\|$.

In order to describe the dielectric response of such a system, one must consider the coupling to a static electric field. Taking the field in the $x$-direction and neglect- ing overlaps between different Wannier functions (tightbinding approximation), we add the coupling term

$$
\hat{H}_{e x t}=-E \hat{X}=-E \sum_{i} x_{i} \hat{n}_{i}
$$

where $\hat{X}$ is the dipole operator (we have put $q=1$ ), $x_{i}$ is the $x$-coordinate of the $i$-th site and $\hat{n}_{i}$ measures the occupation of this site. Here we have assumed that the finite lattice has open boundary conditions, i.e. the connections terminate at the lattice edges. We note that while this is the natural definition for experiments, the notion of response to an applied electric field has recently been generalized to periodic boundary conditions [7]. An applied electric field will induce a polarization at zero temperature given by

$$
P=L^{-d}\langle X\rangle=-L^{-d} \frac{\partial E_{0}}{\partial E}
$$

on a $d$-dimensional lattice with linear dimension $L$, where the average is taken with respect to the ground state of the full Hamiltonian $\hat{H}+\hat{H}_{e x t}$, with corresponding energy $E_{0}$. The zero-field susceptibility is then defined as

$$
\chi=\left.\frac{\partial P}{\partial E}\right|_{E=0}=-\left.L^{-d} \frac{\partial^{2} E_{0}}{\partial E^{2}}\right|_{E=0} .
$$

The examination of the properties of this susceptibility in the vicinity of the Mott metal-insulator transition is the principle aim of this letter.

The susceptibility $\chi$ can be related to the eigenstates $\left|\Psi_{n}\right\rangle$ of $\hat{H}$ using elementary perturbation theory,

$$
\chi=2 L^{-d} \sum_{n \neq 0} \frac{\left|\left\langle\Psi_{0}|\hat{X}| \Psi_{n}\right\rangle\right|^{2}}{\Delta E_{n}},
$$

where $\Delta E_{n}$ is the excitation energy of the $n$-th eigenstate. (Here we have chosen the origin of the coordinate system so that $\langle X\rangle=0$ for $E=0$.) This relation immediately yields a useful inequality in terms of the "charge gap", $\Delta$ (defined as the lowest excitation energy for which the dipole matrix element does not vanish): 


$$
\chi \leq \frac{2}{\Delta} L^{-d}\left\langle\Psi_{0}\left|\hat{X}^{2}\right| \Psi_{0}\right\rangle .
$$

It is thus instructive to consider fluctuations of the polarization, $\left\langle\Psi_{0}\left|\hat{X}^{2}\right| \Psi_{0}\right\rangle$, which can be estimated as follows. We expand the ground state as a series $\left|\Psi_{0}\right\rangle=$ $\sum_{D}\left|\Psi_{0}^{(D)}\right\rangle$, where $D$ is the number of doubly occupied sites ("particles"). At large $U$ the "particles" are located close to empty sites ("holes"). Each particle-hole pair represents an elementary dipole with essentially random orientations. Therefore our estimate is

$$
\left\langle\Psi_{0}\left|\hat{X}^{2}\right| \Psi_{0}\right\rangle=\sum_{D}\left\langle\Psi_{0}^{(D)}\left|\hat{X}^{2}\right| \Psi_{0}^{(D)}\right\rangle \approx\langle D\rangle l^{2},
$$

where $l$ is the average size of the dipoles. Comparing this result with the inequality in Eq. (6), we conclude that a diverging susceptibility requires either a diverging size of the dipoles or a vanishing charge gap or both. In one dimension, the quantity

$$
\xi=\frac{1}{L}\left\langle\Psi_{0}\left|\hat{X}^{2}\right| \Psi_{0}\right\rangle
$$

is a length characterizing the insulating phase [8,9]. We will show below that $\xi$ is the correlation length, up to a dimensionless constant.

On regular lattices, one often faces the problem that the Mott phenomenon, which sets in at large values of $U$ due to charge blocking, is masked by the opening of a charge gap at much lower values of $U$ due to antiferromagnetic order induced by nesting or Umklapp processes. In order to control such effects, we consider here a model that explicitly incorporates frustration of antiferromagnetism, namely the one-dimensional Hubbard model with both nearest-neighbor $t$ and next-nearestneighbor $t^{\prime}$ hopping terms. We set $t=1$ and consider only $t^{\prime} \geq 0$ here because the sign of $t^{\prime}$ is irrelevant at half-filling due to particle-hole symmetry. For $t^{\prime}=0$, the Bethe-Ansatz solution allows one to calculate the charge gap [10], the charge stiffness, and the correlation length in the insulator 11 explicitly. The system is found to be insulating for all positive values of $U$. The metalinsulator transition occurs at $U_{c}=0$ and is infinite order: the charge gap and, correspondingly, the inverse of the correlation length decrease exponentially as $U \rightarrow 0^{+}$. At the same time, the magnetic correlations show a powerlaw decay. For $t^{\prime}>0$, a weak-coupling renormalization group analysis [12] predicts the same behavior as long as there are two Fermi points: Umklapp processes lead to an insulating state for $U>0$, while the magnetic excitation spectrum remains gapless.

For $t^{\prime}>0.5$, there are four Fermi points in the noninteracting band structure and the picture becomes more complicated. In weak coupling, the lowest-order Umklapp processes are marginally irrelevant [12], and the system is predicted to be metallic (vanishing charge gap) with a spin gap. At strong coupling, the model can be mapped to a frustrated Heisenberg chain, which develops a spin gap for $J^{\prime} / J \sim t^{\prime 2}>0.2412$ 13] and incommensurate antiferromagnetic order for $J^{\prime} / J>0.5$. This general picture has been confirmed numerically 14,15 . For a detailed phase diagram, we refer the reader to Fig. 3 of Ref. [15]. Here we will examine both the parameter regime with gapless magnetic excitations and $U_{c}=0\left(t^{\prime} \lesssim 0.5\right)$ and the one with gapped spin degrees of freedom and $U_{c}>0\left(t^{\prime} \gtrsim 0.5\right)$.

In order to numerically evaluate the electric susceptibility, Eq. (4), we use the Density Matrix Renormalization Group (DMRG) 16]. We apply a small electric field so that the system is in a linear response regime (typically $E L=0.001$ ) and measure

$$
\chi=\frac{P}{E}=\frac{1}{L E} \sum_{i} x_{i}\left\langle\hat{n}_{i}\right\rangle
$$

We use the finite-size DMRG algorithm [16, 17] on up to $L=100$ sites, retaining up to 2400 states for the system block. This allows us to keep the sum of the discarded density-matrix eigenvalues to below $10^{-8}$. We have performed extensive tests for $U=0$, a difficult case to treat numerically, and find that we can reproduce analytic results to within less than one percent. The details of the calculations will be described more extensively elsewhere.

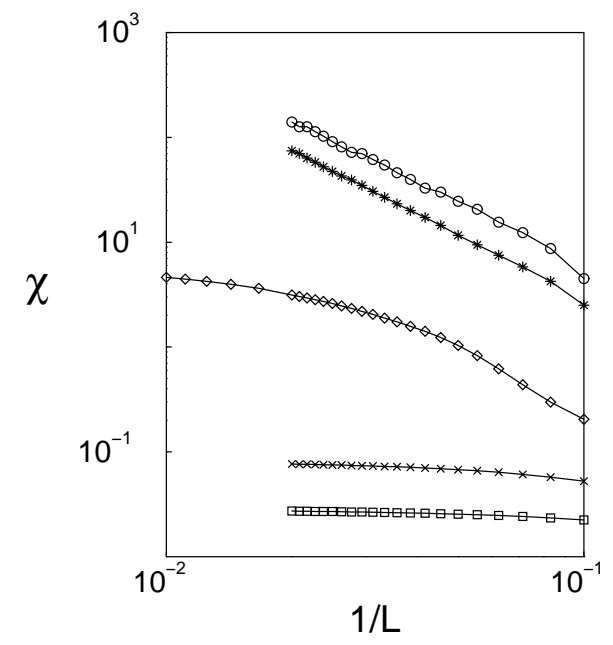

FIG. 1. Electric susceptibility, $\chi$, as a function of $1 / L$ for $U=1$ (circles), $U=2.5$ (stars), $U=4$ (diamonds), $U=5.5$ (crosses), and $U=7$ (squares).

The electric susceptibility $\chi$ is shown in Fig. 1 as a function of the inverse system size for $t^{\prime}=0.7$ and a number of $U$-values. There are two characteristically different behaviors: at small $U$, the system is metallic, and the susceptibility diverges with system size. A fit to a power law in $L$ yields an exponent very close to 2 (within 5\%) for the small $U$ values. For $U=0$, it can be shown analytically that $\chi \sim L^{2}$ for large $L$ for all values of $t^{\prime}$. We conjecture that such a $L^{2}$ divergence of $\chi$ is generic for a one-dimensional perfect metal. For larger 
$U, \chi$ extrapolates to a finite value as $L \rightarrow \infty$. While this is clear for the two larger $U$-values in Fig. 1, care must be taken near the transition because the system appears metallic up to a length scale on the order of the correlation length which diverges at the transition. Such a crossover from metallic to insulating behavior is evident in the $U=4$ curve, for which we have taken lattice sizes of up to $L=100$ to show that $\chi$ scales to a finite value, i.e. that the system is insulating.

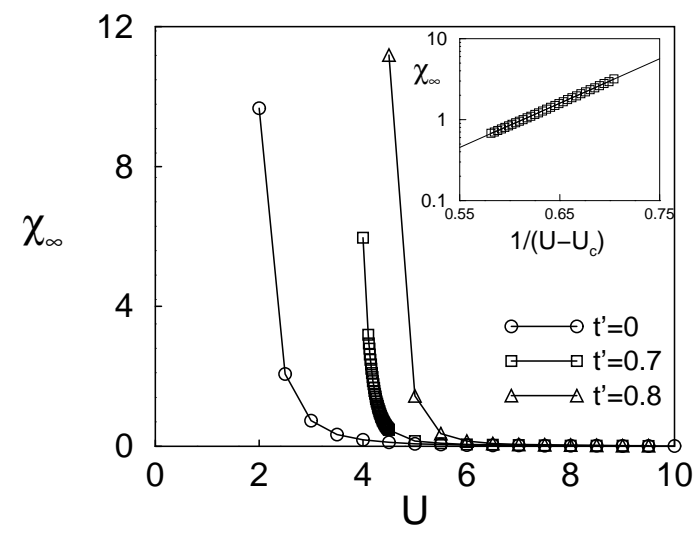

FIG. 2. Electric susceptibility $\chi_{\infty}\left(U, t^{\prime}\right)$ of the infinite-size system for $t^{\prime}=0,0.7,0.8$, as a function of $U$; the lines are guides to the eye. Inset: $\chi_{\infty}\left(U, t^{\prime}=0.7\right)$ for $U=4.1$ to 4.4 (squares) as a function of $1 /\left(U-U_{c}\right)$, on a semilog scale. The line is a fit to an exponential form.

In the insulating regime, we expect $\chi$ to be analytic in $1 / L$. We therefore perform finite-size scaling for large $L$ using a linear fit and extrapolating to $1 / L=0$. The result, $\chi_{\infty}$, is shown in Fig. 2 for $t^{\prime}=0,0.7$, and 0.8 as a function of $U$. For $t^{\prime}=0$, the transition takes place at $U_{c}=0$, as discussed previously. Although we could not obtain a reliable finite-size extrapolation for $U \lesssim 2$ because the correlation length becomes much larger than the system sizes we were able to reach, we could observe numerically that $\chi \sim \Delta^{-2}$ (for $U \lesssim 10$ ), where $\Delta$ is the charge gap given in Ref. [10]. The extrapolation to $\Delta \rightarrow 0$ confirms that $U_{c}=0$. Alternatively, we can fit to the low $-U$ form for $\Delta^{-2}$,

$$
\chi_{\infty}\left(t^{\prime}=0\right)=\frac{A}{U-U_{c}} \exp \left[\frac{B}{\left(U-U_{c}\right)^{\sigma}}\right]
$$

with the exactly known values $B=4 \pi=12.566 \ldots$ and $\sigma=1$; here the prefactor $1 /\left(U-U_{c}\right)$ comes from the logarithmic correction. This yields $U_{c} \simeq 0.058$ and we effectively find that $U_{c}=0$ to within error bars. A fit to the form without the logarithmic correction would yield $U_{c} \simeq 0.209$, which is also consistent with zero, but to within a larger error bar.

It is clear from Fig. 2 that the bigger $t^{\prime}$, the larger the $U$ at which $\chi$ diverges. However, one must perform careful fitting in order to accurately determine $U_{c}$ and the form of the divergence at $t^{\prime}>0$, as an analytical result for the charge gap exists only at $t^{\prime}=0$. For $t^{\prime}=0.7$, we have calculated $\chi$ at many $U$-values near the transition and have fitted to both power law, $\chi \sim\left(U-U_{c}\right)^{\gamma}$ and exponential forms (Eq. 10), but without the logarithmic correction. The logarithmic corrections are, in general, non-universal, i.e. $t^{\prime}$-dependent. Leaving these corrections out, as argued above, will only make the determination of $U_{c}$ less precise. We find that the fit to the power law form yields $U_{c} \simeq 3.4$, a point at which careful finite-size scaling of $\chi$ yields a finite value of $\chi_{\infty}$. Therefore, this $U_{c}$ is clearly too large. The exponential fit yields $\sigma \simeq 1.049, B \simeq 12.45$ and $U_{c} \simeq 2.67$, a more reasonable value of $U_{c}$. Note that the values for $\sigma$ and $B$ are again very close to the ones obtained for $t^{\prime}=0$. The inset of Fig. 2 shows a semilog plot of $\chi_{\infty}$ versus $1 /\left(U-U_{c}\right)$ as well as the fit itself, illustrating its good quality. We therefore find that the exponential form, Eq. (10), expected in an infinite--order transition, characterizes the transition at all $t^{\prime}$, irrespective of whether a spin gap exists or whether $U_{c}$ is finite or zero.

If hyperscaling is valid, there is only one relevant length scale $\xi_{\infty}$ (the correlation length for $L \rightarrow \infty$ ) in the vicinity of the quantum critical point. This length then determines the finite-size scaling of the singular part of the ground state energy density 18]

$$
E_{0}^{\text {sing }} / L^{d}=\xi_{\infty}^{-(d+z)} f\left(L / \xi_{\infty}\right),
$$

where $z$ is the dynamic critical exponent and $f$ a universal scaling function. The quantity $E L$ is an energy and therefore scales like $\xi_{\infty}^{-z}$. Using Eq.(4), one obtains the scaling behavior of the electric susceptibility

$$
\chi=L^{2+z-d} C \Phi\left(L / \xi_{\infty}\right),
$$

where $C$ is a non-universal constant that depends on microscopic details and $\Phi$ is a universal function [19]. The hyperscaling assumption also implies that $\Phi$ tends to a finite value as $L / \xi_{\infty} \rightarrow 0$. This is the region in which the system appears metallic and in which $\chi$ tends to scale like $L^{2}$. Thus $z=1$ is the only consistent value in Eq. (12), in agreement with exact results for $t^{\prime}=0$ [1]. In the opposite limit, $L / \xi_{\infty} \rightarrow \infty$, the system behaves as an insulator for all sizes and $\chi$ tends to a finite value $\chi_{\infty}$. The scaling form (12) with $z=d=1$ thus implies $\lim _{x \rightarrow \infty} \Phi(x)=A / x^{2}$ and

$$
\chi_{\infty}=C A \xi_{\infty}^{2}
$$

where $A$ is a universal constant.

In order to confirm the scaling form Eq.(12) for our model, we plot the DMRG results for $\chi / L^{2}$ as a function of $L / \xi_{\infty}$ in Fig. 3. The quantity $\xi_{\infty}$ is obtained by calculating $\xi$ on finite systems using Eq. (8) and then performing a finite-size extrapolation similar to that used to obtain $\chi_{\infty}$. Notice that all $L$ and $U$ points for a particular $t^{\prime}$ collapse onto the same curve, confirming hyperscaling. Therefore, $\xi_{\infty}$ behaves as the correlation length, 
which we have checked by ascertaining that $\xi_{\infty}$ is the same length (up to a constant) that characterizes the exponential decay of the density-density correlation function.

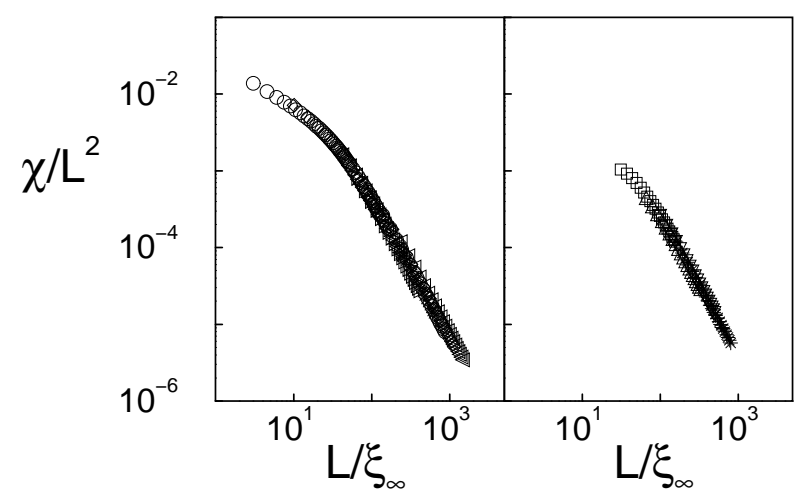

FIG. 3. Scaling plots of $\chi\left(L, U, t^{\prime}\right) / L^{2}$ versus $L / \xi_{\infty}\left(U, t^{\prime}\right)$ in a log-log scale: $t^{\prime}=0$ (left), $t^{\prime}=0.8$ (right). Different symbols correspond to different values of $U$.

The results of the $1 / L$ extrapolation for $\chi$ and $\xi$ are shown in Fig. 1 for three different values of $t^{\prime}$. A power-law fit to $\chi_{\infty}=C^{\prime} \xi_{\infty}^{\tilde{\gamma}}$ yields $\tilde{\gamma}\left(t^{\prime}=0\right) \simeq 1.97$, $\tilde{\gamma}\left(t^{\prime}=0.7\right) \simeq 2.01, \tilde{\gamma}\left(t^{\prime}=0.8\right) \simeq 1.96$, and $C^{\prime}\left(t^{\prime}=\right.$ $0.7) / C^{\prime}\left(t^{\prime}=0\right) \simeq 1, C^{\prime}\left(t^{\prime}=0.8\right) / C^{\prime}\left(t^{\prime}=0\right) \simeq 0.7$. This confirms the scaling behavior (13). It also shows that the constant $C$ depends weakly on $t^{\prime}$.

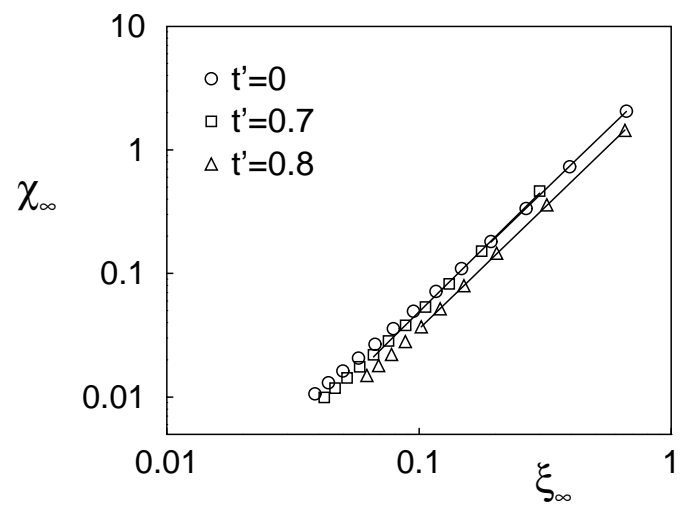

FIG. 4. Electric susceptibility $\chi_{\infty}$, versus correlation length $\xi_{\infty}$ for different values of $t^{\prime}$. The lines are power-law fits.

In summary, our calculations for the $U-t-t^{\prime}$ chain at half-filling confirm that the electric susceptibility $\chi$ (and therefore also the dielectric constant $\varepsilon=1+4 \pi \chi$ ) diverge when approaching the Mott transition from the insulating side. The polarization fluctuations, which also diverge for $U \rightarrow U_{c}$ from above, have been found to be directly proportional to the correlation length $\xi$ of the Mott insulating phase. In agreement with the hyperscaling hypothesis, the metallic or insulating behavior of the finite-size system depends only on the ratio $L / \xi_{\infty}$. The finite-size scaling of $\chi$ can then be related to a universal scaling function and a dynamic exponent $z=1$. The transition is found to be infinite order and to show the same critical behavior whether there is a spin gap or not. As to the origin of this "dielectric catastrophe", we conclude, on the basis of both the inequality $\chi \leq 2 \xi / \Delta$ and the observed scaling $\chi_{\infty} \sim \xi_{\infty}^{2}$, that it involves both a diverging correlation length $\xi$ (linked to the unbinding of dipoles) and a vanishing of the charge gap $\Delta$.

We thank the Swiss National Foundation for financial support, under Grant No. 20-53800.98. Parts of the numerical computations were done at the Swiss Center for Scientific Computing. We thank F.F. Assaad, S. Daul, E. Jeckelmann, G.I. Japaridze, S. Sachdev, C.A. Stafford and $\mathrm{X}$. Zotos for valuable discussions.

[1] W. Kohn, Phys. Rev. 133, A171 (1964).

[2] N. F. Mott, Metal-Insulator Transitions, Taylor and Francis, London (1974).

[3] T. T. Rosenbaum et al., Phys. Rev. B 27, 7509 (1983).

[4] P. W. Anderson, in Frontiers and Borderlines in ManyParticle Physics, eds. R. A. Broglia and J. R. Schrieffer, North Holland, Amsterdam (1988), p.1.

[5] F. Gebhard, The Mott Metal-Insulator Transition, Springer Tracts in Modern Physics, Vol. 137 (1997).

[6] S. Sachdev, Quantum Phase Transitions, Cambridge University Press, 1999.

[7] R. Resta, Phys. Rev. Lett. 80, 1800 (1998); R. Resta and S. Sorella, Phys. Rev. Lett. 82, 370 (1999).

[8] E. K. Kudinov, Sov. Phys. Solid State 33, 1299 (1991).

$[9]$ I. Souza, T. Wilkins, and R. M. Martin, condmat/9911007 (to be published).

[10] A. A. Ovchinnikov, Sov. Phys. JETP 30, 1160 (1970).

[11] C. A. Stafford and A. J. Millis, Phys. Rev. B 48, 1409 (1993).

[12] M. Fabrizio, Phys. Rev. B 54, 10054 (1996).

[13] S. Eggert, Phys. Rev. B 54, 9612 (1996); K. Okamoto and K. Nomura, Phys. Lett. A 169, 422 (1992).

[14] R. Arita, K. Kuroki, H. Aoki, and M. Fabrizio, Phys. Rev. B 57, 10324 (1998).

[15] S. Daul and R. M. Noack, Phys. Rev. B 61, 1646 (2000).

[16] S. R. White, Phys. Rev. Lett. 69, 2863 (1992); Phys. Rev. B 48, 10345 (1993).

[17] R. M. Noack and S. R. White, in Density Matrix Renormalization: A New Numerical Method in Physics, edited by I. Peschel et al. (Springer Verlag, Berlin, 1999).

[18] K. Kim and P. B. Weichman, Phys. Rev. B 43, 13583 (1991).

[19] V. Privman and M. E. Fisher, Phys. Rev. B 30, 322 (1984). 